\title{
Pump Frequency Resonances for Light-Induced Incipient Superconductivity in $\mathrm{YBa}_{2} \mathrm{Cu}_{3} \mathrm{O}_{6.5}$
}

\author{
B. Liu, ${ }^{1}$ M. Först, ${ }^{1}$ M. Fechner, ${ }^{1}$ D. Nicoletti®,${ }^{1}$ J. Porras, ${ }^{3}$ T. Loew, ${ }^{3}$ B. Keimer, ${ }^{3}$ and A. Cavalleri ${ }^{1,2, *}$ \\ ${ }^{1}$ Max Planck Institute for the Structure and Dynamics of Matter, 22761 Hamburg, Germany \\ ${ }^{2}$ Department of Physics, University of Oxford, Clarendon Laboratory, Oxford OX1 3PU, United Kingdom \\ ${ }^{3}$ Max Planck Institute for Solid State Research, 70569 Stuttgart, Germany
}

(Received 19 May 2019; revised manuscript received 13 January 2020; accepted 27 January 2020; published 3 March 2020)

\begin{abstract}
Optical excitation in the cuprates has been shown to induce transient superconducting correlations above the thermodynamic transition temperature $T_{C}$, as evidenced by the terahertz-frequency optical properties in the nonequilibrium state. In $\mathrm{YBa}_{2} \mathrm{Cu}_{3} \mathrm{O}_{6+x}$, this phenomenon has so far been associated with the nonlinear excitation of certain lattice modes and the creation of new crystal structures. In other compounds, like $\mathrm{La}_{2-x} \mathrm{Ba}_{x} \mathrm{CuO}_{4}$, similar effects were reported also for excitation at near-infrared frequencies, and were interpreted as a signature of the melting of competing orders. However, to date, it has not been possible to systematically tune the pump frequency widely in any one compound, to comprehensively compare the frequency-dependent photosusceptibility for this phenomenon. Here, we make use of a newly developed nonlinear optical device, which generates widely tunable high-intensity femtosecond pulses, to excite $\mathrm{YBa}_{2} \mathrm{Cu}_{3} \mathrm{O}_{6.5}$ throughout the entire optical spectrum (3-750 THz). In the far-infrared region (3-24 THz), signatures of nonequilibrium superconductivity are induced only for excitation of the 16.4- and 19.2-THz vibrational modes that drive $c$-axis apical oxygen atomic positions. For higher driving frequencies $(25-750 \mathrm{THz})$, a second resonance is observed around the charge transfer band edge at approximately $350 \mathrm{THz}$. These findings highlight the importance of coupling to the electronic structure of the $\mathrm{CuO}_{2}$ planes, mediated either by a phonon or by charge transfer.

DOI: $10.1103 /$ PhysRevX.10.011053

Subject Areas: Condensed Matter Physics, Nonlinear Dynamics, Superconductivity
\end{abstract}

The equilibrium superconducting state of high- $T_{C}$ cuprates manifests itself in a number of characteristic features in the terahertz-frequency optical response. In Fig. 1, we report selected optical properties measured in $\mathrm{YBa}_{2} \mathrm{Cu}_{3} \mathrm{O}_{6.5}$ above and below the superconducting transition temperature $T_{C}$.

As the temperature is lowered from 100 (black curve, $T \gg T_{C} \simeq 52 \mathrm{~K}$ ) to $10 \mathrm{~K}$ (red curve, $T \ll T_{C}$ ), the real part of the $c$-axis optical conductivity, $\sigma_{1}(\omega)$, evolves from that of a semiconductor with thermally activated carriers to a gapped spectrum [see Fig. 1(b)1]. Simultaneously, a zerofrequency $\delta$ peak emerges, indicative of dissipationless dc transport. This peak is not seen directly in $\sigma_{1}(\omega)$ but is reflected in an $1 / \omega$ divergence in the imaginary conductivity, $\sigma_{2}(\omega)$ [Fig. 1(b)2]. Correspondingly, a sharp edge in the optical reflectivity of the superconducting state develops

*andrea.cavalleri@mpsd.mpg.de

Published by the American Physical Society under the terms of the Creative Commons Attribution 4.0 International license. Further distribution of this work must maintain attribution to the author(s) and the published article's title, journal citation, and DOI. Funded by the Max Planck Society. at the Josephson plasma resonance (JPR) $\omega_{\mathrm{JPR}} \simeq 30 \mathrm{~cm}^{-1}$ [4-6] [Fig. 1(b)3].

A number of recent pump-probe experiments have shown that these same optical signatures [red curves in Fig. 1(b)] can be recreated transiently in $\mathrm{YBa}_{2} \mathrm{Cu}_{3} \mathrm{O}_{6+x}$ for base temperatures $T \gg T_{C}$ by optical excitation made resonant with the $20-\mathrm{THz}$ lattice vibrations that modulate the position of the apical oxygen atoms along the $c$ axis [13]. Measurements of the transient atomic structure with femtoscond $\mathrm{x}$-ray diffraction revealed an average structural deformation in $\mathrm{YBa}_{2} \mathrm{Cu}_{3} \mathrm{O}_{6.5}$ [7], associated with nonlinear lattice dynamics $[8,9]$. It was reasoned that such a transient structure may favor higher-temperature superconductivity [7].

However, the response of $\mathrm{YBa}_{2} \mathrm{Cu}_{3} \mathrm{O}_{6+x}$ or that of any other material has never been systematically checked for excitation of different lattice modes, as no optical device existed that could generate high-intensity pulses with sufficient spectral selectivity and tunability throughout the terahertz spectrum.

Furthermore, recent work in single-layer cuprates of the type $\mathrm{La}_{2-x} \mathrm{Ba}_{x} \mathrm{CuO}_{4}$ [10-14] has evidenced transient optical properties similar to those observed for mid-infrared driving in $\mathrm{YBa}_{2} \mathrm{Cu}_{3} \mathrm{O}_{6+x}$, for excitation in the near-infrared and 
(a)

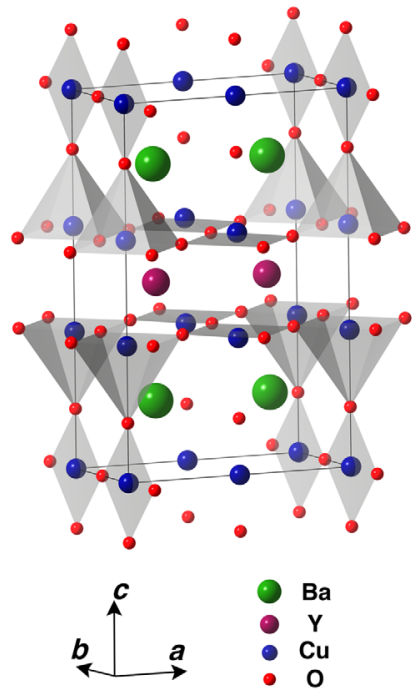

(b)

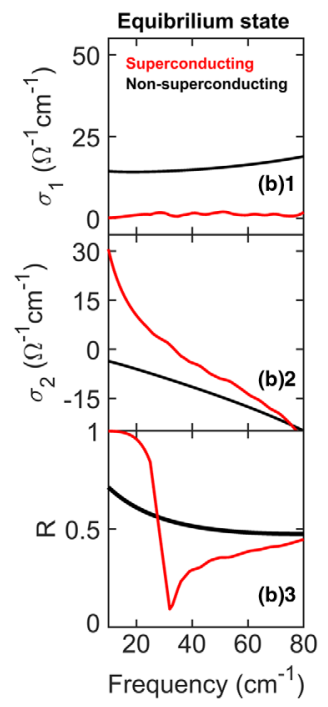

FIG. 1. Crystal structure and equilibrium terahertz optical properties of $\mathrm{YBa}_{2} \mathrm{Cu}_{3} \mathrm{O}_{6.5}$. (a) Crystallographic unit cell of orthorhombic $\mathrm{YBa}_{2} \mathrm{Cu}_{3} \mathrm{O}_{6.5}$. Bilayers of conducting $\mathrm{CuO}_{2}$ planes, stacked along the $c$ direction, form Josephson junctions in the superconducting state. (b) $c$-axis terahertz-frequency optical properties in the equilibrium superconducting $\left(T<T_{C}\right.$, red curves) and normal ( $T>T_{C}$, black curves) state. The real $\left[\sigma_{1}(\omega)\right]$ and imaginary $\left[\sigma_{2}(\omega)\right]$ parts of the optical conductivity are displayed along with the normal-incidence reflectivity $R(\omega)$ (the same data as those reported in Refs. [1-3]).

visible part of the spectrum, a phenomenon that has been assigned to melting of a competing charge order $[15,16]$ (as already discussed for $\mathrm{La}_{1.675} \mathrm{Eu}_{0.2} \mathrm{Sr}_{0125} \mathrm{CuO}_{4}$ [17]).

Here, we report a comprehensive study of the response of $\mathrm{YBa}_{2} \mathrm{Cu}_{3} \mathrm{O}_{6.5}$ (the same compound investigated in Refs. $[1-3,7])$ to excitation at all frequencies throughout the terahertz electromagnetic spectrum (3-24 THz; $\left.100-800 \mathrm{~cm}^{-1}\right)$, as well as in the near-infrared and visible range (up to $750 \mathrm{THz}$ ). To this end, we make use of a newly developed nonlinear optical device [18] based on difference frequency mixing of chirped near-infrared pulses [19] in organic crystals (see Supplemental Material [20]).

In a first set of experiments, we studied the response to excitation of all phonons in the far infrared (3-24 THz; $100-800 \mathrm{~cm}^{-1}$ ). The relevant pump frequency range is displayed in Fig. 2(b), which reports the equilibrium broadband $c$-axis optical conductivity $\sigma_{1}(\omega)$ at $T=$ $100 \mathrm{~K}[5,6]$.

Here, we show data taken under the same conditions as those reported in Refs. [1-3], to be used as a reference point for all the experiments that follow. The excitation pulses were centered at $19.2 \mathrm{THz}\left(640 \mathrm{~cm}^{-1}\right)$, which correspond to apical oxygen distortions at the oxygendeficient chains. These experiments were performed with the same broadband 4-THz-wide pulses used in Refs. [1-3] and then, for comparison, with the newly available $1-\mathrm{THz}$ spectral bandwidth (narrow band). These pump pulses,

polarized along the $c$ axis of a $\mathrm{YBa}_{2} \mathrm{Cu}_{3} \mathrm{O}_{6.5}$ single crystal [20], were focused onto the sample at a fluence of approximately $8 \mathrm{~mJ} / \mathrm{cm}^{2}$. Note that in the narrow-band experiments the pulses were 4 times longer than in the broadband experiments (600 fs vs $150 \mathrm{fs}$ ). As these two measurements used the same pump fluence, the peak electric fields were of 3 and $6 \mathrm{MV} / \mathrm{cm}$ for narrow-band (long pulse) and broadband (short pulse) excitation, respectively.

As already discussed in Refs. [1-3], the transient $c$-axis optical properties were interrogated between 15 and $80 \mathrm{~cm}^{-1}$ by reflecting a second terahertz probe pulse generated by optical rectification in a nonlinear crystal. The electric field of these pulses, after reflection from the sample surface, was electro-optically sampled [Fig. 2(b), gray spectrum] for different pump-probe time delays (see Supplemental Material [20] for further details).

Figures 2(c) and 2(d) report the photoinduced changes in the complex optical conductivity at $T=100 \mathrm{~K} \gg T_{C}$, as a function of the frequency and pump-probe time delay (color plots). Both excitation schemes induced qualitatively similar optical properties, with a significant increase in the imaginary conductivity $\sigma_{2}(\omega)$, which became positive and exhibited a superconductinglike $1 / \omega$ divergence for $\omega \rightarrow 0$.

This increase is particularly evident in the frequency spectra measured at the peak of the signal, displayed above the color plots in Figs. 2(c) and 2(d) (right). In these line cuts, the transient $\sigma_{2}(\omega)$ measured at $\tau \simeq 0.5 \mathrm{ps}$ after excitation (blue dots) is superimposed with the equilibrium $\sigma_{2}(\omega)$ at $T<T_{C}$ (red line) for comparison.

Note that in both these experiments, for which the excitation fluence was a factor of 2 higher than that reported in Refs. [1-3], the response was that of a homogeneous medium. The effective medium model, introduced to reproduce the partial changes in the optical properties in Refs. [1,2], was no longer necessary to explain the data. We interpret this observation by positing that the excitation range achieved with the new setup may have overcome a dynamical "percolation threshold."

Strikingly, for narrow-band excitation at $T=100 \mathrm{~K}$, we observed exactly the same $\sigma_{2}(\omega)$ spectrum measured in the equilibrium superconducting state $\left(T=10 \mathrm{~K} \ll T_{C}=52 \mathrm{~K}\right)$ in the same sample.

The photoinduced dynamics at longer time delays ( $\tau \gtrsim 1 \mathrm{ps)}$ evidenced decoherence and increased dissipation, as observed in the real part of the optical conductivity, $\sigma_{1}(\omega)$ [Figs. 2(c) and 2(d), left]. On the left-hand side of each panel, we plot two frequency-integrated quantities as a function of the time delay: $\left.\omega \sigma_{2}(\omega)\right|_{\omega \rightarrow 0}$, which in an equilibrium superconductor is proportional to the superfluid density, and $\int \Delta \sigma_{1}(\omega) d \omega$, which is a reporter of dissipation and quasiparticle heating [21]. For both broadband and narrow-band excitation, it is evident that the dissipative part of the optical response $\left[\int \Delta \sigma_{1}(\omega) d \omega\right.$, left 


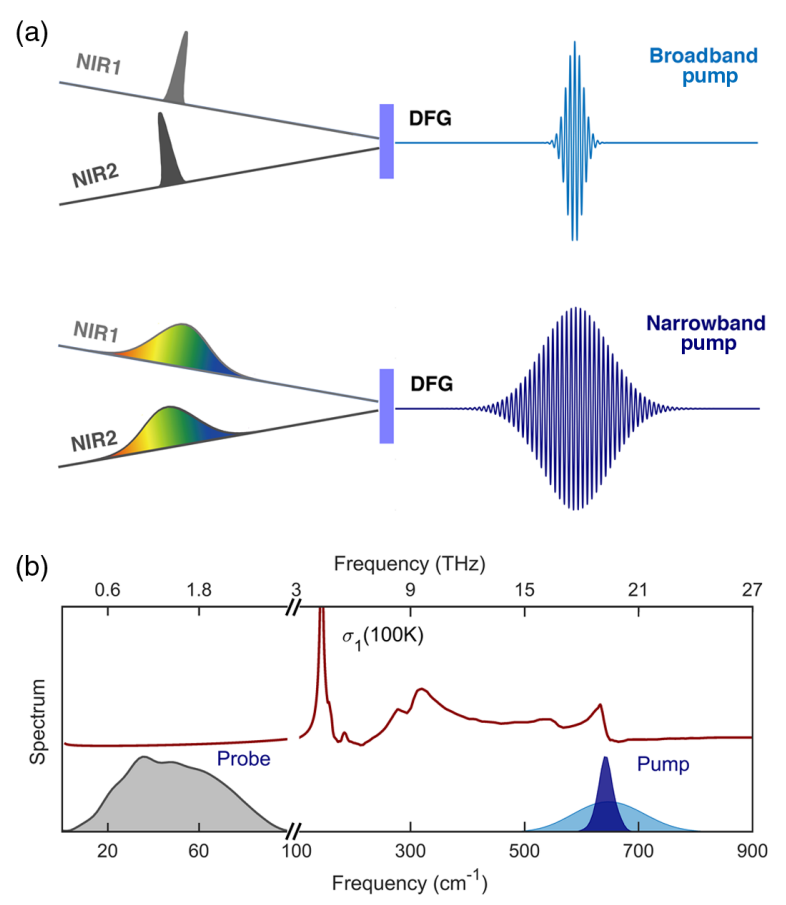

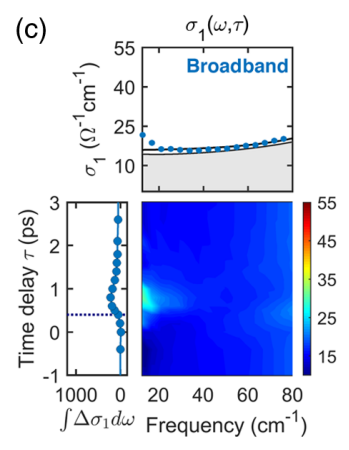

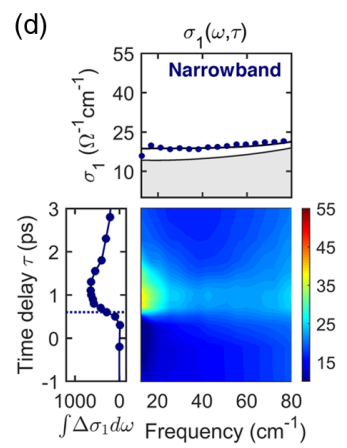

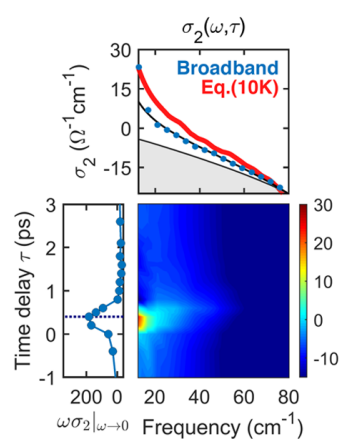

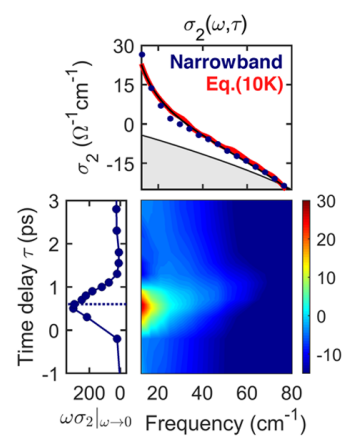

FIG. 2. Transient $c$-axis optical conductivity induced by apical oxygen excitation above $T_{C}$. (a) Schematics of the generation processes for broadband and narrow-band excitation pulses. The signal outputs (NIR1 and NIR2) of two parallel optical parametric amplifiers were directly sent to a nonlinear crystal to generate broadband pump pulses (relative bandwidth $\Delta \nu / \nu \sim 20 \%$ ) in a difference frequency generation (DFG) process. For narrow-band generation $(\Delta \nu / \nu<10 \%)$, the two signal outputs were linearly chirped before the DFG process (see Supplemental Material [20] for further details). (b) Equilibrium $c$-axis optical conductivity $\sigma_{1}(\omega)$ of $\mathrm{YB}_{2} \mathrm{Cu}_{3} \mathrm{O}_{6.5}$ at $100 \mathrm{~K}$ ( $T>T_{C}$, red solid line), along with the frequency spectra of the terahertz probe (gray), broadband pump (light blue), and 19.2-THz narrowband pump (dark blue) pulses. (c) Color plots: Frequency- and time-delay-dependent complex optical conductivity measured after broadband excitation at $T=100 \mathrm{~K}$. Upper: Corresponding $\sigma_{1}(\omega)$ and $\sigma_{2}(\omega)$ line cuts displayed at equilibrium (gray lines) and at the peak of the coherent response ( $\tau \simeq 0.5 \mathrm{ps}$ time delay, blue circles). Black lines are fits to the transient spectra with a model describing the response of a Josephson plasma (see Supplemental Material [20] for details on the fitting procedure). For comparison, we also report the equilibrium $\sigma_{2}(\omega)$ measured in the superconducting state at $T=10 \mathrm{~K}$ (red line). Side: Frequency-integrated dissipative $\left[\int \Delta \sigma_{1}(\omega) d \omega\right]$ and coherent $\left[\left.\omega \sigma_{2}(\omega)\right|_{\omega \rightarrow 0}\right]$ responses, as a function of the pump-probe time delay. The delay corresponding to the spectra reported in the top $(\tau \simeq 0.5 \mathrm{ps})$ is indicated by a dashed line. (d) The same quantities as in (c), measured for narrow-band excitation at $19.2 \mathrm{THz}$.

panels in Figs. 2(c) and 2(d)], increases only at later time delays compared to the superconducting component, reaching a maximum after the peak in $\left.\omega \sigma_{2}(\omega)\right|_{\omega \rightarrow 0}$ has relaxed [right in Figs. 2(c) and 2(d)].

Figure 3 reports a more comprehensive set of experiments, obtained by tuning the pump pulse frequency widely throughout the far-infrared spectrum. Four selected results are displayed, corresponding to resonant narrow-band excitation of four different phonon modes (see Supplemental Material [20] for additional datasets). The data reported in Fig. $2\left(\omega_{\text {pump }}=19.2 \mathrm{THz}=\right.$ $640 \mathrm{~cm}^{-1}$ ) are shown alongside the results for excitations at $\omega_{\text {pump }}=16.4 \mathrm{THz}=547 \mathrm{~cm}^{-1}, \omega_{\text {pump }}=10.1 \mathrm{THz}=$ $337 \mathrm{~cm}^{-1}$, and $\omega_{\text {pump }}=4.2 \mathrm{THz}=140 \mathrm{~cm}^{-1}$, all driven by maintaining constant $3 \mathrm{MV} / \mathrm{cm}$ peak electric field strength.

The atomic displacements of these vibrational modes are displayed in Fig. 3(a). The 4.2-THz mode involves motions of the barium atoms and of the apical oxygens, while the
10.1-THz mode is associated with a planar $\mathrm{Cu}-\mathrm{O}$ buckling motion. The 16.4- and 19.2-THz modes, whose individual responses to photoexcitation could not be separated with the previously available broadband pump pulses [1-3], involve almost exclusively oscillations of the apical oxygen atoms on oxygen-rich and oxygen-deficient $\mathrm{Cu}-\mathrm{O}$ chains, respectively.

The four pump-probe experiments reported in Figs. 3(b) and 3(c), where we display selected spectra taken at the peak of the coherent response (at time delay $\tau \simeq 0.5 \mathrm{ps}$ ), show that driving at the two highest-frequency modes (16.4 and $19.2 \mathrm{THz}$ ) induces a superconductinglike response $\left[\sigma_{2}(\omega) \propto 1 / \omega\right]$, for which the transient complex conductivity is fitted by a model describing the optical response of a Josephson plasma. On the other hand, excitation of the two low-frequency modes ( 4.2 and $10.1 \mathrm{THz}$ ) causes only a moderate increase in dissipation and no superconducting component. This observation could be well reproduced, for all time delays, by a Drude-Lorentz model for normal 

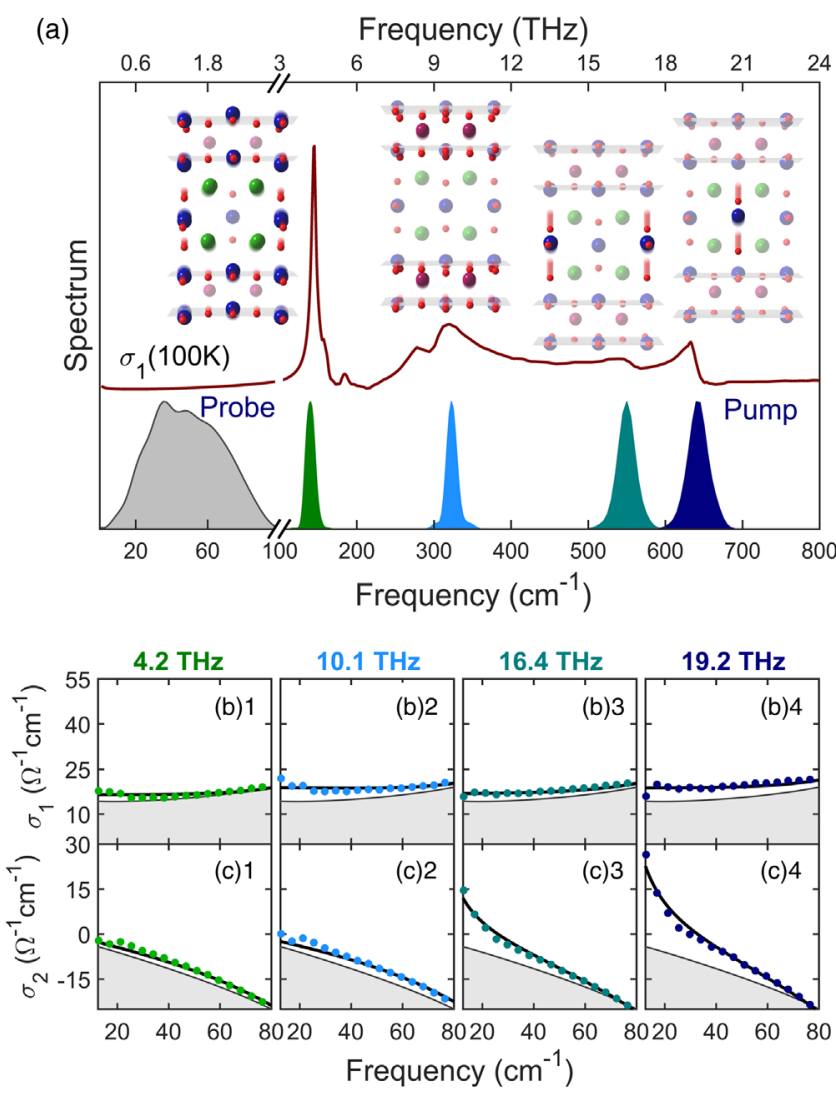

FIG. 3. Transient $c$-axis optical response induced by modeselective phonon excitations above $T_{C}$. (a) Equilibrium $c$-axis optical conductivity $\sigma_{1}(\omega)$ of $\mathrm{YB}_{2} \mathrm{Cu}_{3} \mathrm{O}_{6.5}$ at $100 \mathrm{~K}\left(T>T_{C}\right.$, red solid line), along with the frequency spectra of the terahertz probe (gray) and of different narrow-band pump pulses, tuned to be resonant with four different optical phonons at 4.2, 10.1, 16.4, and 19.2 THz. The insets sketch the atomic motions related to each of these modes, with $\mathrm{CuO}_{2}$ layers highlighted in gray and transparency applied to quasistationary atoms (see Fig. 1 for the atom labeling). (b),(c) Complex optical conductivity $\sigma_{1}(\omega)+$ $i \sigma_{2}(\omega)$ measured before (gray lines) and at $\tau \simeq 0.5 \mathrm{ps}$ time delay (colored circles) after resonant stimulation of the phonon modes shown in (a) with $8 \mathrm{~mJ} / \mathrm{cm}^{2}$ pump fluence (corresponding to approximately $3 \mathrm{MV} / \mathrm{cm}$ peak electric field). The black solid lines are fits to the transient spectra performed with either a simple Drude-Lorentz model for normal conductors [(b)1, (b)2, (c)1, (c)2] or a model describing the response of a Josephson plasma [(b)3, (b)4, (c)3, (c)4] (see Supplemental Material [20] for more details on the fitting procedure).

conductors (see Supplemental Material [20] for details on the fitting procedure).

The same experiments as those reported in Fig. 3 were systematically repeated for 42 pump frequencies throughout the far-infrared spectrum (3-24 THz), for which we report in Fig. 4 the results of the analysis of the transient optical properties at the time delay corresponding to the peak of the coherent response.

In Fig. 4(a), we show the total, spectrally integrated probe signal, that is, the modulus of the complex optical

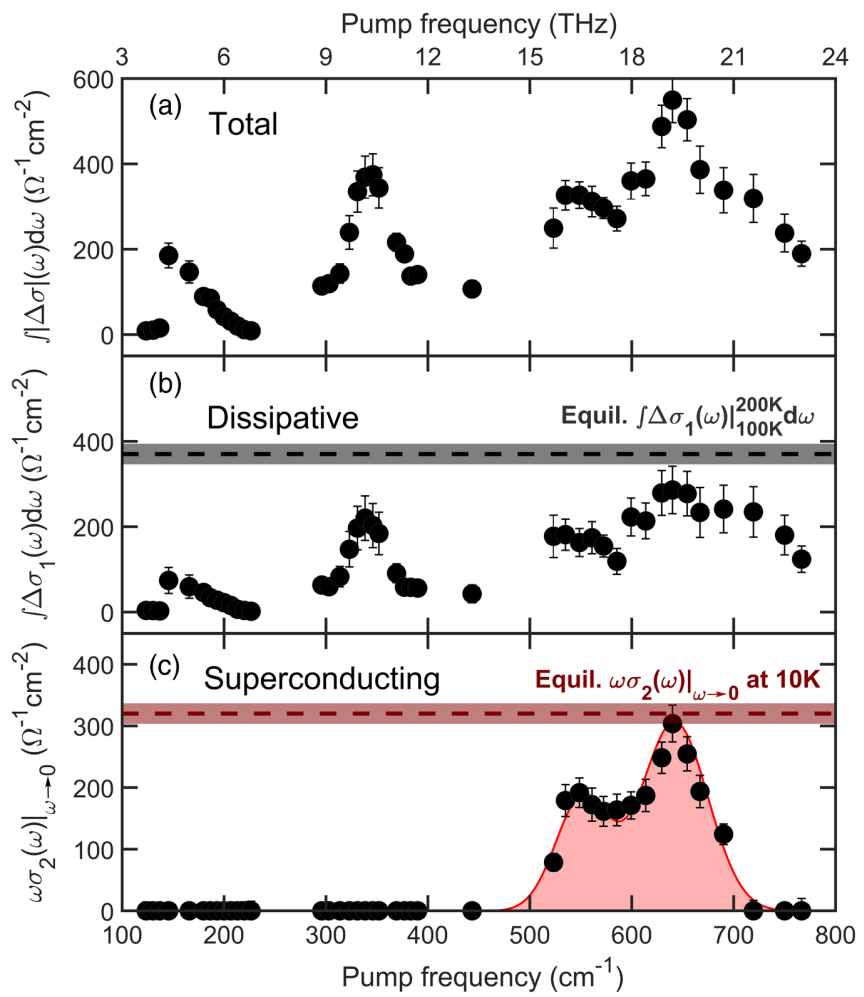

FIG. 4. Evolution of the photoinduced response at $T=100 \mathrm{~K}$ as a function of the excitation frequency. Distinct quantities, extracted from the transient optical conductivity at $\tau \simeq 0.5 \mathrm{ps}$ time delay, are displayed as a function narrow-band excitation frequency: (a) the total, frequency integrated change in optical conductivity $\int|\Delta \sigma(\omega)| d \omega$, (b) the frequency integrated dissipative response $\int \Delta \sigma_{1}(\omega) d \omega$, and (c) the superconducting response represented by the low-frequency limit $\left.\omega \sigma_{2}(\omega)\right|_{\omega \rightarrow 0}$. The red shaded region represents the frequency range around the apical oxygen phonon modes, where a transient superconductinglike response could be identified. Horizontal dashed lines indicate the thermally induced increase in $\int \sigma_{1}(\omega) d \omega$ when heating the sample from 100 to $200 \mathrm{~K}$ (black) and the equilibrium superfluid density $\left.\omega \sigma_{2}(\omega)\right|_{\omega \rightarrow 0}$ measured at $T=10 \mathrm{~K}$ (red). All data were taken with a pump fluence of $8 \mathrm{~mJ} / \mathrm{cm}^{2}$ (corresponding to approximately $3 \mathrm{MV} / \mathrm{cm}$ peak electric field).

conductivity. In Fig. 4(b), we display only the dissipative component of the signal $\left[\int \Delta \sigma_{1}(\omega) d \omega\right]$ and in Fig. 4(c) only the superconducting contribution $\left.\omega \sigma_{2}(\omega)\right|_{\omega \rightarrow 0}$ (see Supplemental Material [20] for extended datasets).

For comparison, we have also included horizontal dashed lines indicating the thermally induced increase in $\int \sigma_{1}(\omega) d \omega$ when heating the sample from 100 to $200 \mathrm{~K}$ [Fig. 4(b)] and the equilibrium superfluid density $\left.\omega \sigma_{2}(\omega)\right|_{\omega \rightarrow 0}$, measured at $T=10 \mathrm{~K}$ [Fig. 4(c)].

For excitation at 19.2 and $16.4 \mathrm{THz}$, the nonequilibrium state includes a dissipative $\int \Delta \sigma_{1}(\omega) d \omega$ response (analogous to that observed upon heating) that coexists with a superconductinglike imaginary conductivity $\left[\left.\omega \sigma_{2}(\omega)\right|_{\omega \rightarrow 0}\right]$ identical to that measured in the same material in the equilibrium superconducting state. 

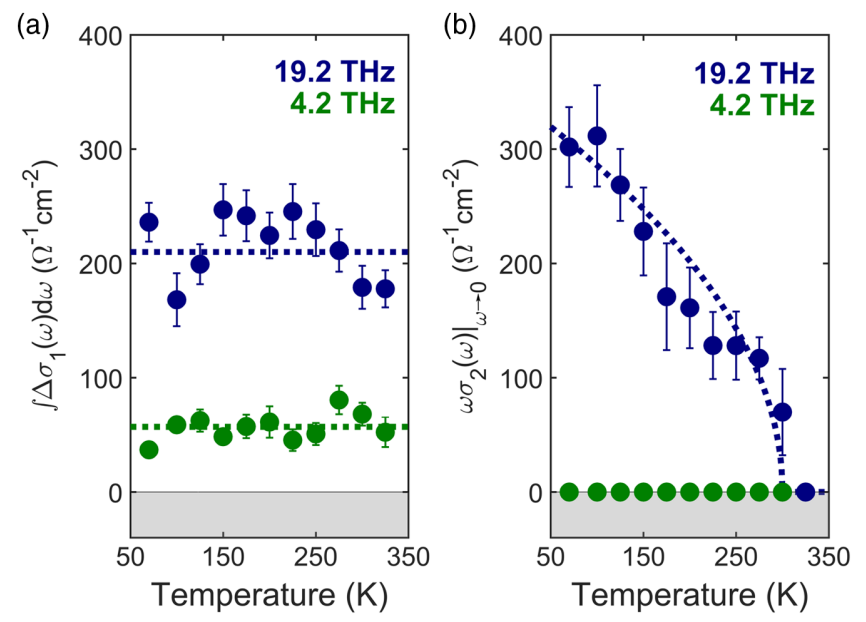

FIG. 5. Temperature dependence of the dissipative and superconductinglike responses. Temperature-dependent (a) dissipative response $\int \Delta \sigma_{1}(\omega) d \omega$ and (b) superconductinglike response $\left.\omega \sigma_{2}(\omega)\right|_{\omega \rightarrow 0}$, measured for resonant narrow-band excitation of the lowest-frequency $(4.2 \mathrm{THz})$ and highest-frequency (19.2 THz) phonon modes. The dashed lines are guides to the eye. As in Fig. 4, all data were taken with a pump fluence of $8 \mathrm{~mJ} / \mathrm{cm}^{2}$ (corresponding to approximately $3 \mathrm{MV} / \mathrm{cm}$ peak electric field).

On the other hand, when the pump frequency is tuned below $15 \mathrm{THz}\left(500 \mathrm{~cm}^{-1}\right)$, no superconductinglike component is observed, whereas the dissipative response is approximately the same as that observed for $\omega_{\text {pump }}>15 \mathrm{THz}$.

The different nature of the dissipative and the superconductinglike signal is underscored by the data reported in Fig. 5. Here, we show the dependence of $\int \Delta \sigma_{1}(\omega) d \omega$ and $\left.\omega \sigma_{2}(\omega)\right|_{\omega \rightarrow 0}$ on the base temperature $\left(T>T_{C}\right)$, for two different pump frequencies.

The dissipative term [Fig. 5(a)] is temperature independent and persists all the way up to $325 \mathrm{~K}$, which suggests that its origin may be related to heating of quasiparticles. On the other hand, the superconductinglike response [Fig. 5(b)], which is observed only for highfrequency pumping (blue circles), displays a strong reduction with increasing temperature, almost disappearing for $T>300 \mathrm{~K}$. This result is consistent with the observation reported in Refs. [2,3].

Additional datasets taken in the superconducting state at $T<T_{C}$ are reported in the Supplemental Material [20]. There, we show that the preexisting, equilibrium superfluid density can be transiently enhanced (in agreement with Refs. [1,2]) only by photoexcitation at $19.2 \mathrm{THz}$, provided that a sufficiently high pump fluence is employed. For weaker driving fields [22], or when the pump is detuned to lower frequencies, only a transient depletion of the superconducting condensate is observed.

In a second set of experiments, we studied the response of the material to excitation at higher frequencies, above the
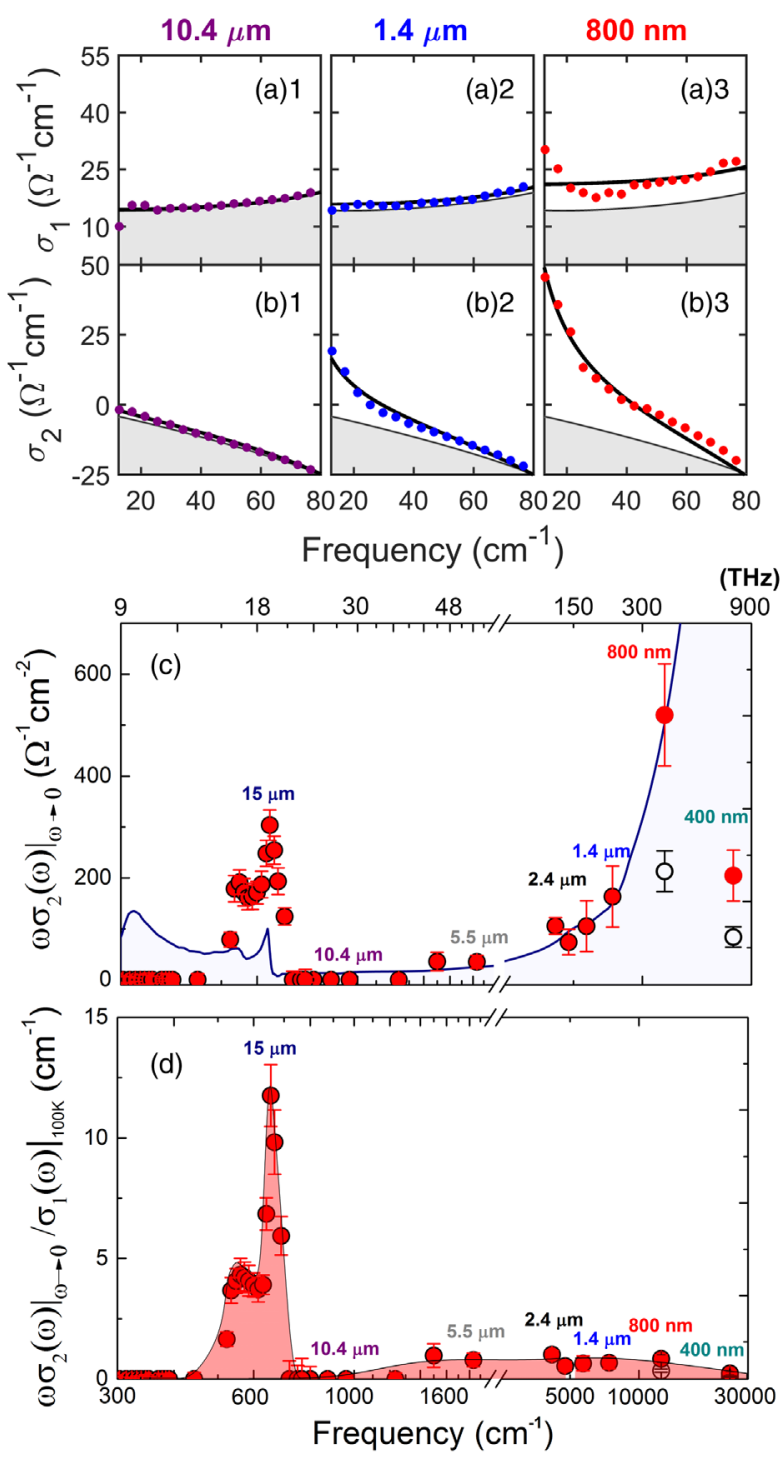

FIG. 6. Transient $c$-axis optical response induced by high-energy charge excitation at $T=100 \mathrm{~K}$. (a),(b) Complex optical conductivity $\sigma_{1}(\omega)+i \sigma_{2}(\omega)$, measured at equilibrium (gray) and at $\tau \simeq$ $0.5 \mathrm{ps}$ time delay (colored circles) after excitation at three different pump wavelengths with approximately $8 \mathrm{~mJ} / \mathrm{cm}^{2}$ pump fluence. The black solid lines are fits to the transient spectra performed with either a simple Drude-Lorentz model for normal conductors [(a)1, (b)1] or a model describing the response of a Josephson plasma [(a) 2, (b)2, (a)3, (b)3] (see Supplemental Material [20] for more details on the fitting procedure). (c) Pump frequency dependence of the superconducting response, represented by the low-frequency limit $\left.\omega \sigma_{2}(\omega)\right|_{\omega \rightarrow 0}$ (red circles). The equilibrium optical conductivity $\sigma_{1}^{\text {equil }}\left(\omega_{\text {pump }}\right)$ is shown as a blue line. (d) Normalized response $\left.\omega \sigma_{2}(\omega)\right|_{\omega \rightarrow 0} / \sigma_{1}^{\text {equil }}\left(\omega_{\text {pump }}\right)$ (red circles). The gray line is a multiLorentzian fit. All data for $\omega_{\text {pump }}<300 \mathrm{THz}$ were taken with constant pump fluence $\left(8 \mathrm{~mJ} / \mathrm{cm}^{2}\right)$ and peak electric field $(3 \mathrm{MV} / \mathrm{cm})$ at a fixed pulse duration of $600 \mathrm{fs}$. At $\lambda_{\text {pump }}=$ $800 \mathrm{~nm}$ and $\lambda_{\text {pump }}=400 \mathrm{~nm}$, for which the pump pulses were $100 \mathrm{fs}$ long, we report data points taken at both $8 \mathrm{~mJ} / \mathrm{cm}^{2}$ (approximately $7 \mathrm{MV} / \mathrm{cm}$, red circles) and $1.5 \mathrm{~mJ} / \mathrm{cm}^{2}$ (approximately $3 \mathrm{MV} / \mathrm{cm}$, empty circles), keeping either the excitation fluence or peak electric field at the same values. 
phonon resonances and up to the region where electronic bands are found.

In Figs. 6(a) and 6(b), we report similar measurements as those shown in Fig. 3 and display the transient complex conductivity at the peak of the light-induced response for three representative excitation frequencies: $29 \mathrm{THz}\left(\lambda_{\text {pump }}=\right.$ $10.4 \mu \mathrm{m}), 214 \mathrm{THz}\left(\lambda_{\text {pump }}=1.4 \mu \mathrm{m}\right)$, and $375 \mathrm{THz}\left(\lambda_{\text {pump }}=\right.$ $800 \mathrm{~nm})$.

Similar to what is reported in Fig. 4 for frequencies immediately above $19 \mathrm{THz}$, the response at $29 \mathrm{THz}\left(\lambda_{\text {pump }}=\right.$ $10.4 \mu \mathrm{m}$ ) displays no superconductinglike component.

However, for excitation frequencies of $214 \mathrm{THz}\left(\lambda_{\text {pump }}=\right.$ $1.4 \mu \mathrm{m})$ and $375 \mathrm{THz}\left(\lambda_{\text {pump }}=800 \mathrm{~nm}\right)$, a transient $\sigma_{2}(\omega) \propto 1 / \omega$ is observed again, resembling that induced by driving resonant with the apical oxygen phonons.

Note that, for these higher frequencies, the reconstruction procedure is less reliable than for the experiments reported in Figs. 2-5, as the penetration depth mismatch of pump and probe pulses becomes larger. Indeed, at all times, the raw response (optically induced change of the reflected terahertz probe electric field) is largest at the two phonon resonances displayed in Figs. 3 and 4 [20]. The error bars for high excitation frequencies [see Fig. 6(c)] reflect this uncertainty. Nevertheless, an error analysis indicates that the appearance of a divergent imaginary conductivity is robust.

A complete pump frequency dependence for the superconducting component $\left.\omega \sigma_{2}(\omega)\right|_{\omega \rightarrow 0}$ is displayed in Fig. 6(c) (see Supplemental Material [20] for extended datasets). A negligible response of the imaginary conductivity is found for all driving frequencies between the apical oxygen phonon (approximately $21 \mathrm{THz}$ ) and $42 \mathrm{THz}$, whereas for higher pump frequencies a second resonance emerges, with a strength that follows the increase in pump absorption on the charge transfer resonance [visualized in this figure by the equilibrium optical conductivity $\left.\sigma_{1}^{\text {equil }}\left(\omega_{\text {pump }}\right)\right]$.

Figure 6(d) displays the same data after normalization against the oscillator strength of the material at each pump frequency. The normalized quantity $\left.\omega_{\text {probe }} \sigma_{2}\left(\omega_{\text {probe }}\right)\right|_{\omega \rightarrow 0} /$ $\sigma_{1}^{\text {equil }}\left(\omega_{\text {pump }}\right)$ is the frequency-dependent "photosusceptibility" for the transient state and, therefore, to be taken as an alternative way of visualizing the efficiency of the effect.

In the aggregate, the body of work reported above prompts the following considerations. First, from the data in Figs. 3-5, it is clear that some form of mode-specific lattice excitation must underpin optically induced superconductivity in the terahertz frequency range. In previous studies [7,9], it was conjectured that the lowest-order nonlinear lattice anharmonicity, of the type $Q_{I R}^{2} Q_{R}$ (where $Q_{I R}$ and $Q_{R}$ are the normal coordinates of the directly driven infrared-active mode and any anharmonically coupled Raman mode, respectively [8]), may explain the observed phenomenology. Indeed, this lattice term leads to a transient, average structural deformation that may be beneficial to superconductivity [7].
Hence, in addition to the nonlinear phononic mechanism, which is validated by $\mathrm{x}$-ray experiments and certainly present, other phenomena are likely to come into play. As documented in the Supplemental Material [20], when considering the calculated average lattice deformations induced by an anharmonic $Q_{I R}^{2} Q_{R}$ coupling for driving each of the $I R$ phonons in Fig. 3, one does not find a defining feature for the two modes at 16.4 and $19.2 \mathrm{THz}$.

Note also that the two high-frequency vibrations drive large-amplitude motions of the apical oxygen atoms, which are then expected to couple directly to the in-plane electronic and magnetic structure. This coupling is supposed to be much weaker for the other modes at a lower frequency $[23,24]$. We also note that the frequency of the two apical oxygen phonons matches approximately the sum of the inter- and intrabilayer Josephson plasma frequencies in $\mathrm{YBa}_{2} \mathrm{Cu}_{3} \mathrm{O}_{6.5} \quad\left(\omega_{\text {JPR }, 1} \simeq 1-2 \mathrm{THz}\right.$ and $\omega_{\mathrm{JPR}, 2} \simeq 14 \mathrm{THz}$, respectively). Hence, a mechanism in which driven lattice excitations couple directly to the inplane electronic structure may become resonantly enhanced at these frequencies [25-29].

The second resonance found at the charge transfer band appears to reinforce the notion that changes in the electronic properties of the planes are key in the observed phenomenon [30,31]. At the doping level studied here, charge-order melting is not expected to play an important role, as for $\mathrm{YBa}_{2} \mathrm{Cu}_{3} \mathrm{O}_{6.6}$ [32] and $\mathrm{La}_{1.875} \mathrm{Ba}_{0.125} \mathrm{CuO}_{4}$ [15]. At these frequencies, for the excitation polarized along the $c$ axis, we expect a rearrangement of the electronic structure with some qualitative analogy to the direct action of the apical oxygen modes. It is, therefore, possible that a similar mechanism to that responsible for the resonances at 19.2 and $16.4 \mathrm{THz}$ is at play. We have noted that for the two apical oxygen modes parametric excitation of interlayer fluctuations would be resonant with the sum frequency of the intra- and interbilayer modes [29]. Here, a similar parametric coupling may be at play, without the frequency resonance and, hence, less efficient.

Clearly, further studies that make use of the new pump device available here are needed, with special attention to measurements of time-dependent lattice dynamics [7] and inelastic excitations [33-35]. More generally, the tunable, spectrally selective nonlinear pump source, applied for the first time in the present study, is expected to strongly impact the investigation of nonequilibrium phenomena in solids.

\section{ACKNOWLEDGMENTS}

The research leading to these results received funding from the European Research Council under the European Union's Seventh Framework Programme (FP7/2007-2013)/ERC Grant Agreement No. 319286 (QMAC). We acknowledge support from the Deutsche Forschungsgemeinschaft (DFG) via the Cluster of Excellence 'The Hamburg Centre for Ultrafast Imaging' (EXC 1074_Project ID 194651731) and the priority program SFB925. 
[1] W. Hu, S. Kaiser, D. Nicoletti, C. R. Hunt, I. Gierz, M. C. Hoffmann, M. Le Tacon, T. Loew, B. Keimer, and A. Cavalleri, Optically Enhanced Coherent Transport in $\mathrm{YBa}_{2} \mathrm{Cu}_{3} \mathrm{O}_{6.5}$ by Ultrafast Redistribution of Interlayer Coupling, Nat. Mater. 13, 705 (2014).

[2] S. Kaiser, C. R. Hunt, D. Nicoletti, W. Hu, I. Gierz, H. Y. Liu, M. Le Tacon, T. Loew, D. Haug, B. Keimer, and A. Cavalleri, Optically Induced Coherent Transport far above $T_{c}$ in Underdoped $\mathrm{YBa}_{2} \mathrm{Cu}_{3} \mathrm{O}_{6+\delta}$, Phys. Rev. B 89, 184516 (2014).

[3] C. R. Hunt, D. Nicoletti, S. Kaiser, D. Pröpper, T. Loew, J. Porras, B. Keimer, and A. Cavalleri, Dynamical Decoherence of the Light Induced Interlayer Coupling in $\mathrm{YBa}_{2} \mathrm{Cu}_{3} \mathrm{O}_{6+\delta}$, Phys. Rev. B 94, 224303 (2016).

[4] D. Basov and T. Timusk, Electrodynamics of High-T Superconductors, Rev. Mod. Phys. 77, 721 (2005).

[5] C. C. Homes, T. Timusk, D. A. Bonn, R. Liang, and W. N. Hardy, Optical Properties along the c-Axis of $\mathrm{YBa}_{2} \mathrm{Cu}_{3} \mathrm{O}_{6+x}$ for $x=0.50 \rightarrow 0.95$ Evolution of the Pseudogap, Physica (Amsterdam) 254C, 265 (1995).

[6] C. C. Homes, T. Timusk, D. A. Bonn, R. Liang, and W. N. Hardy, Optical Phonons Polarized along the c-Axis of $\mathrm{YBa}_{2} \mathrm{Cu}_{3} \mathrm{O}_{6+x}$, for $x \rightarrow 0.5$ to 0.95, Can. J. Phys. 73, 663 (1995).

[7] R. Mankowsky, A. Subedi, M. Först, S. O. Mariager, M. Chollet, H. Lemke, J. Robinson, J. Glownia, M. Minitti, A. Frano, M. Fechner, N. A. Spaldin, T. Loew, B. Keimer, A. Georges, and A. Cavalleri, Nonlinear Lattice Dynamics as a Basis for Enhanced Superconductivity in $\mathrm{YBa}_{2} \mathrm{Cu}_{3} \mathrm{O}_{6.5}$, Nature (London) 516, 71 (2014).

[8] M. Först, C. Manzoni, S. Kaiser, Y. Tomioka, Y. Tokura, R. Merlin, and A. Cavalleri, Nonlinear Phononics as an Ultrafast Route to Lattice Control, Nat. Phys. 7, 854 (2011).

[9] R. Mankowsky, M. Först, T. Loew, J. Porras, B. Keimer, and A. Cavalleri, Coherent Modulation of the $\mathrm{YBa}_{2} \mathrm{Cu}_{3} \mathrm{O}_{6+x}$ Atomic Structure by Displacive Stimulated Ionic Raman Scattering, Phys. Rev. B 91, 094308 (2015).

[10] D. Nicoletti, E. Casandruc, Y. Laplace, V. Khanna, C. R. Hunt, S. Kaiser, S. S. Dhesi, G. D. Gu, J. P. Hill, and A. Cavalleri, Optically-Induced Superconductivity in Striped $\mathrm{La}_{2-x} \mathrm{Ba}_{x} \mathrm{CuO}_{4}$ by Polarization-Selective Excitation in the Near Infrared, Phys. Rev. B 90, 100503(R) (2014).

[11] E. Casandruc, D. Nicoletti, S. Rajasekaran, Y. Laplace, V. Khanna, G. D. Gu, J. P. Hill, and A. Cavalleri, WavelengthDependent Optical Enhancement of Superconducting Interlayer Coupling in $\mathrm{La}_{1.885} \mathrm{Ba}_{0.115} \mathrm{CuO}_{4}$, Phys. Rev. B 91, 174502 (2015).

[12] D. Nicoletti, D. Fu, O. Mehio, S. Moore, A. S. Disa, G. D. $\mathrm{Gu}$, and $\mathrm{A}$. Cavalleri, Magnetic-Field Tuning of LightInduced Superconductivity in Striped $\mathrm{La}_{2-x} \mathrm{Ba}_{x} \mathrm{CuO}_{4}$, Phys. Rev. Lett. 121, 267003 (2018).

[13] K. A. Cremin, J. Zhang, C. C. Homes, G. D. Gu, Z. Sun, M. M. Fogler, A. J. Millis, D. N. Basov, and R. D. Averitt, Photo-Enhanced Metastable c-Axis Electrodynamics in Stripe Ordered Cuprate $\mathrm{La}_{1.885} \mathrm{Ba}_{0.115} \mathrm{CuO}_{4}$, Proc. Natl. Acad. Sci. U.S.A. 116, 19875 (2019).

[14] S. J. Zhang, Z. X. Wang, L. Y. Shi, T. Lin, M. Y. Zhang, G. D. Gu, T. Dong, and N. L. Wang, Light-Induced New Collective Modes in $\mathrm{La}_{1.905} \mathrm{Ba}_{0.095} \mathrm{CuO}_{4}$ Superconductor, Phys. Rev. B 98, 020506(R) (2018).
[15] M. Först, R. I. Tobey, H. Bromberger, S. B. Wilkins, V. Khanna, A. D. Caviglia, Y. D. Chuang, W. S. Lee, W. F. Schlotter, J. J. Turner, M. P. Minnitti, O. Krupin, X. J. Xu, J. S. Wen, G. D. Gu, S. S. Dhesi, A. Cavalleri, and J.P. Hill, Melting Charge Stripes in Vibrationally Driven $\mathrm{La}_{1.875} \mathrm{Ba}_{0.125} \mathrm{CuO}_{4}$ : Assessing the Respective Roles of Electronic and Lattice Order in Frustrated Superconductors, Phys. Rev. Lett. 112, 157002 (2014).

[16] V. Khanna, R. Mankowsky, M. Petrich, H. Bromberger, S. A. Cavill, E. Möhr-Vorobeva, D. Nicoletti, Y. Laplace, G. D. Gu, J.P. Hill, M. Först, A. Cavalleri, and S. S. Dhesi, Restoring Interlayer Josephson Coupling in $\mathrm{La}_{1.885} \mathrm{Ba}_{0.115} \mathrm{CuO}_{4}$ by Charge Transfer Melting of Stripe Order, Phys. Rev. B 93, 224522 (2016).

[17] D. Fausti, R. I. Toby, N. Dean, S. Kaiser, A. Dienst, M. Hoffmann, S. Pyon, T. Takayam, H. Takagi, and A. Cavalleri, Light Induced Superconductivity in a Striped Ordered Cuprate, Science 331, 189 (2011).

[18] B. Liu, H. Bromberger, A. Cartella, T. Gebert, M. Först, and A. Cavalleri, Generation of Narrowband High-Intensity, Carrier-Envelope Phase Stable Pulses Tunable between 4 and $18 \mathrm{THz}$, Opt. Lett. 42, 129 (2017).

[19] A. Cartella, T. F. Nova, A. Oriana, G. Cerullo, M. Först, C. Manzoni, and A. Cavalleri, Narrowband Carrier-Envelope Phase Stable Mid-infrared Pulses at Wavelengths beyond $10 \mu \mathrm{m}$ by Chirped-Pulse Difference Frequency Generation, Opt. Lett. 42, 663 (2017).

[20] See Supplemental Material at http://link.aps.org/ supplemental/10.1103/PhysRevX.10.011053 for details on the experimental setup, data acquisition and evaluation, equilibrium optical properties, uncertainties in the determination of the transient optical properties, fitting models, extended datasets, pump electric field dependence, transient response below $T_{C}$, pump-induced heating, and the $a b$ initio calculations of the structural dynamics.

[21] M. Dressel and G. Grüner, Electrodynamics of Solids (Cambridge University Press, Cambridge, England, 2002).

[22] S. J. Zhang, Z. X. Wang, H. Xiang, X. Yao, Q. M. Liu, L. Y. Shi, T. Lin, T. Dong, D. Wu, and N. L. Wang, Photoinduced Nonequilibrium Response in Underdoped $\mathrm{YBa}_{2} \mathrm{Cu}_{3} \mathrm{O}_{6+x}$ Probed by Time-Resolved Terahertz Spectroscopy, arXiv:1904.10381 [Phys. Rev. X (to be published)].

[23] M. Mori, G. Khalliullin, T. Tohyama, and S. Maekawa, Origin of the Spatial Variation of the Pairing Gap in Bi-Based High Temperature Cuprate Superconductors, Phys. Rev. Lett. 101, 247003 (2008).

[24] Y. Y. Peng, G. Dellea, M. Conni, A. Amorese, D. Di Castro, G. M. DeLuca, K. Kummer, M. Salluzzo, X. Sun, X. J. Zhou, G. Balestino, M. LeTacon, B. Keimer, L. Braicovic, N. B. Brookes, and G. Ghiringhelli, Influence of Apical Oxygen on the Extent of the In-Plane Exchange Interaction in Cuprate Superconductors Nat. Phys. 13, 1201 (2017).

[25] S. J. Denny, S. R. Clark, Y. Laplace, A. Cavalleri, and D. Jaksch, Proposed Parametric Cooling of Bilayer Cuprate Superconductors by Terahertz Excitation, Phys. Rev. Lett. 114, 137001 (2015).

[26] J. I. Okamoto, A. Cavalleri, and L. Mathey, Theory of Enhanced Interlayer Tunneling in Optically Driven High $T_{c}$ Superconductors, Phys. Rev. Lett. 117, 227001 (2016). 
[27] M. Knap, M. Babadi, G. Refael, I. Martin, and E. Demler, Dynamical Cooper Pairing in Nonequilibrium Electron-Phonon Systems, Phys. Rev. B 94, 214504 (2016).

[28] M. Babadi, M. Knap, I. Martin, G. Refael, and E. Demler, Theory of Parametrically Amplified Electron-Phonon Superconductivity, Phys. Rev. B 96, 014512 (2017).

[29] A. von Hoegen, M. Fechner, M. Först, J. Porras, B. Keimer, M. Michael, E. Demler, and A. Cavalleri, Probing Coherent Charge Fluctuations in $\mathrm{YBa}_{2} \mathrm{Cu}_{3} \mathrm{O}_{6+x}$ at Wavevectors outside the Light Cone, arXiv:1911.08284.

[30] G. Yu, C. H. Lee, A. J. Heeger, N. Herron, E. M. McCarron, L. Cong, G. C. Spalding, C. A. Nordman, and A. M. Goldman, Phase Separation of Photogenerated Carriers and Photoinduced Superconductivity in High- $T_{C}$ Materials, Phys. Rev. B 45, 4964 (1992).

[31] G. Nieva, E. Osquiguil, J. Guimpel, M. Maenhoudt, B. Wuyts, Y. Bruynseraede, M. B. Maple, and I. K. Schuller, Photoinduced Enhancement of Superconductivity, Appl. Phys. Lett. 60, 2159 (1992).
[32] M. Först, A. Frano, S. Kaiser, R. Mankowsky, C. R. Hunt, J. J. Turner, G. L. Dakovski, M. P. Minitti, J. Robinson, T. Loew, M. Le Tacon, B. Keimer, J. P. Hill, A. Cavalleri, and S. S. Dhesi, Femtosecond X Rays Link Melting of ChargeDensity Wave Correlations and Light-Enhanced Coherent Transport in $\mathrm{YBa}_{2} \mathrm{Cu}_{3} \mathrm{O}_{6.6}$, Phys. Rev. B 90, 184514 (2014).

[33] M. Buzzi, M. Först, R. Mankowsky, and A. Cavalleri, Probing Dynamics in Quantum Materials with Femtosecond X-Rays, Nat. Rev. Mater. 3, 299 (2018).

[34] L. J. P. Ament, M. van Veenendaal, T. P. Devereaux, J. P. Hill, and J. van den Brink, Resonant Inelastic X-Ray Scattering Studies of Elementary Excitations, Rev. Mod. Phys. 83, 705 (2011).

[35] H.-H. Kim, S. M. Souliou, M. E. Barber, E. Lefrançois, M. Minola, M. Tortora, R. Heid, N. Nandi, R. A. Borzi, G. Garbarino, A. Bosak, J. Porras, T. Loew, M. König, P. J. W. Moll, A. P. Mackenzie, B. Keimer, C. W. Hicks, and M. Le Tacon, Uniaxial Pressure Control of Competing Orders in a High-Temperature Superconductor, Science 362, 1040 (2018). 